POS PROCEEDINGS

\title{
Overview of the GRB observation by POLAR
}

\author{
Shaolin Xiong ${ }^{*}$, Yuanhao Wang ${ }^{a b}$, Zhengheng Li ${ }^{a b}$, Jianchao Sun ${ }^{a}$, Yi Zhao ${ }^{a}$, \\ Hancheng $\mathbf{L i}{ }^{a b}$, Yue Huang ${ }^{a}$, on behalf of the POLAR collaboration ${ }^{\dagger}$ \\ ${ }^{a}$ Key Laboratory of Particle Astrophysics, Institute of High Energy Physics, Beijing 100049, \\ China \\ ${ }^{b}$ University of Chinese Academy of Sciences, Beijing 100049, China \\ E-mail: xiongsleihep.ac.cn
}

POLAR is a dedicated Compton scattering polarimeter to detect Gamma-Ray Bursts (GRBs) in the energy range of about 50 to $500 \mathrm{keV}$, which was launched on-board the Chinese space laboratory Tiangong-2 (TG2) on Sep 15, 2016. Since then, POLAR has detected 55 GRBs jointly with other telescopes as of Apr 1, 2017. Meanwhile, more GRBs detected by POLAR only will be revealed as the burst search and identification program gets more sophisticated to deal with the complicated background. After subtracting the non-observation period due to varies reasons in the commissioning phase, the effective observation time is about 3180 hours, thus the GRB detection rate of POLAR is estimated to be about $150 \mathrm{GRBs} / \mathrm{year}$, demonstrating that POLAR is one of the most sensitive GRB detectors in operation. Thanks to the high sensitivity, large FOV (about $1 / 2$ of the all-sky) and unique capability of polarization measurement, POLAR is a very important detector in the search for high-energy electromagnetic counterpart for Gravitational Wave Bursts (GWB), High Energy Neutrinos (HEN) and Fast Radio Bursts (FRB). In this paper, we summarized the GRB observation by POLAR, including GRB search and identification, joint observation and Gamma-ray Coordinates Network (GCN) alerts, data analysis, GRB catalogues and so on.

35th International Cosmic Ray Conference - ICRC2017

10-20 July, 2017

Bexco, Busan, Korea

\footnotetext{
*Speaker.

${ }^{\dagger}$ Abous us at our website: polar.ihep.ac.cn
} 


\section{Introduction}

Gamma-Ray Bursts (GRBs) are the most violent explosions in the cosmos since the big bang. They are classified into two types according to the observed duration of the X/gamma-ray emission (dubbed prompt emission) [1]: Short GRBs, which have duration less than $2 \mathrm{~s}$, are believed to originate from the coalescence of two compact objects, such as neutron stars and neutron star, or neutron star and black hole, whereas the long GRBs (duration longer than $2 \mathrm{~s}$ ) are thought to generate by the collapse of massive star. According to the current theoretical models, the prompt high-energy emission are radiated from the relativistic jets launched by the central engine which is usually a black hole or a massive, fast rotating magnetar. In contrast to the short-lived, high energy prompt emission, the afterglow emission lasts much longer but usually spans in lower energies (soft X-ray, optical and radio band, but also seen in GeV occasionally), which is produced by the collision of the jets with interstellar medium (ISM) around the progenitor of the GRB [2].

Despite of nearly a half century of observation and research, the GRBs still remain mystery with many open questions in the jet composition, radiation mechanism, central engine and progenitor [2]. Many competing theoretical models can interpret the current observed temporal and spectral features of the GRBs. Therefore, new observations, especially the polarization measurements, are required to distinguish these models [3].

POLAR is a dedicated GRB polarimeter sensitive in $50-500 \mathrm{keV}$, aiming to measure the polarization degree and polarization angle for a large sample of GRBs with an unprecedented accuracy. It consists of 25 detector modules, each of which employs an array of 64 plastic scintillators read out by a multi-anodes photomultiplier (MAPMT). More information about POLAR mission and its preliminary science results could be found in $[4,5,6,7]$.

Due to the limited telemetry resources for POLAR, there is a latency of about 5 to 20 hours for the POLAR Science Data Center (PSDC) to receive the observation data. After downlinked, the data will be synchronized as soon as possible between the PSDC in the Institute of High Energy Physics in China, and the University of Geneva and the Paul Scherrer Institut (PSI) in Switzerland within the POLAR collaboration.

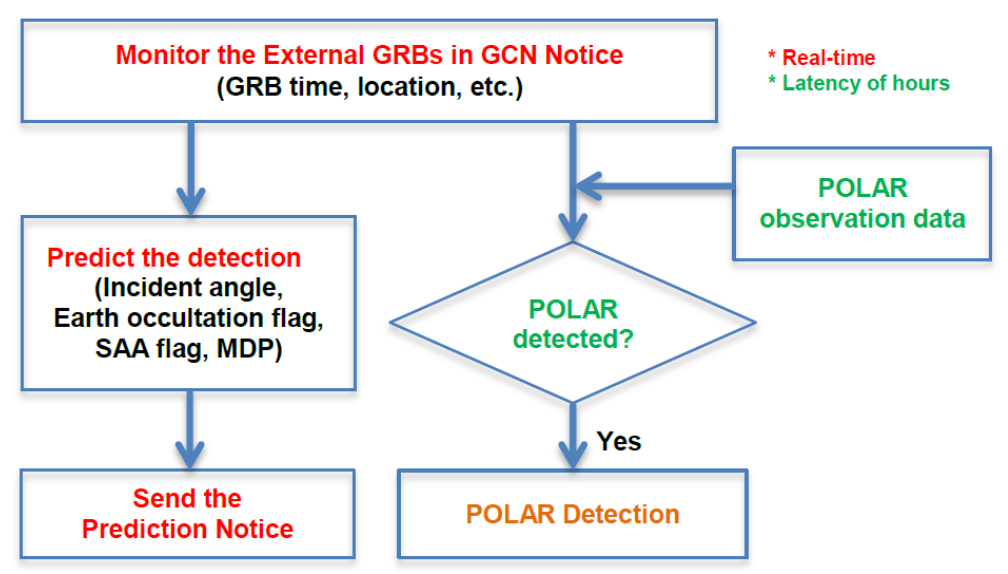

Figure 1: flow chart for external triggers 


\section{GRB search and identification}

Since there is no real-time data downlink, POLAR cannot provide real-time triggers for GRBs. Nevertheless, in order to maximize the GRB science return, we implemented two approaches for POLAR to give the GRB detection information as soon as possible. The first approach is initiated by external GRBs reported in GCN Notice by other telescopes, while the second one is triggered by the ground search of the POLAR data. They will be presented in details in following sections.

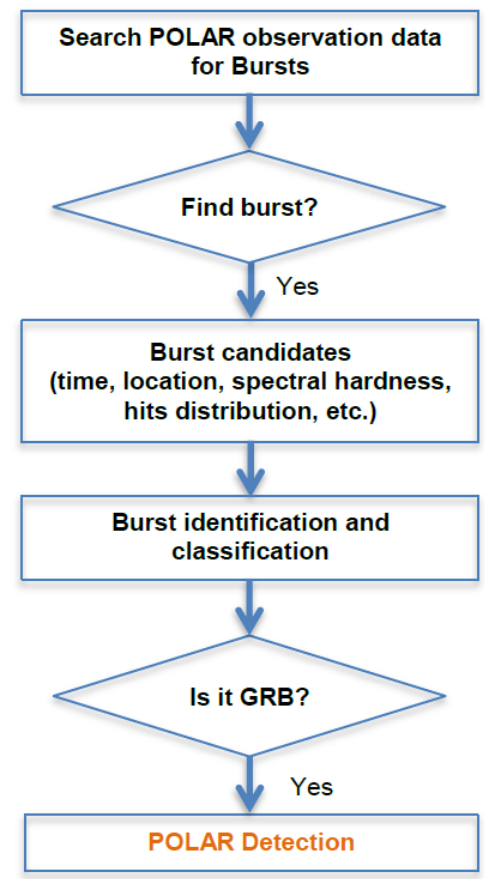

Figure 2: flow chart for internal triggers

\subsection{External triggers}

Many telescopes report GRBs with GCN Notice in (nearly) real-time, which we called external triggers or external GRBs. But POLAR data suffers hours of delay, this prevents us from checking, in real time, whether POLAR detect the external GRBs or not. However, since the attitude and orbit of POLAR is well predictable according to the operation plan, we can predict whether and how well POLAR would detect the external GRBs immediately after receiving the GCN Notice. Such prompt prediction information might be useful in the follow-up observations; therefore we plan to share it with the GRB community by sending the POLAR prediction GCN notice.

To do so, we developed software to automatically monitor external GRBs reported in GCN Notice and calculate whether the GRB fall inside the FoV of POLAR. In addition, based on the fluence and duration of the GRB, the incident angle to POLAR, and the background rate of POLAR, we further estimate how well POLAR will measure the polarization of the GRB, i.e. Minimum Detectable Polarization (MDP). 
When the POLAR observation data come in, we directly check the data around the time of the external GRB and compare the real observation to the prediction mentioned above. As of Apr 1, 2017, POLAR have detected 55 GRBs (Table 3) using this approach.

\subsection{Internal triggers}

POLAR is able to monitor GRBs occurred in all sky unblocked by the Earth and the TG2 spacecraft, thus we expect POLAR can not only detect GRBs jointly with other telescopes, but also discover new GRBs not found by any others. We developed the burst search program to blind search the POLAR data for GRBs, i.e. internal triggers or internal GRBs. Just like other GRB missions, the search algorithm is to find out the sources above the fluctuation of background. The search is implemented for 8 timescales (i.e. $64 \mathrm{~ms}, 128 \mathrm{~ms}, 256 \mathrm{~ms}, 1.024 \mathrm{~s}, 2.048 \mathrm{~s}, 4.096 \mathrm{~s}$ and $8.192 \mathrm{~s}$ ) with 4 phases for each timescale, and for different energy ranges.

According to the pre-flight simulation, the majority of the in-flight background comes from the charged particles in the space environment. Due to the relatively high inclination angle of POLAR (43 degree), the in-flight background variation is quite complicated with significant amount of bumps and spikes probably caused by the space environment. These non-GRB events are difficult to distinguish from GRBs just based on the light curve, therefore, in order to efficiently identify and classify GRBs from these non-GRB events, joint criteria, which include the spacecraft location, spectral hardness, hits distribution in 1600 bars and localization of the burst, is required. Such criteria are not easy to establish due to the elusive low-energy particle events in the orbit. We expect to find out more GRBs in POLAR data once the search and identification program get more sophisticated.

\section{GRB analysis}

For every GRB detected by POLAR, a series of analysis is planned to do by the Burst Advocate (BA), as shown in Fig. 3. The first analysis is to produce the background-subtracted light curve and calculate the duration (T90), peak rate and total counts for this burst. BA is responsible to publish these analysis by sending out a GCN Circular. To date, 49 Circulars have been published by POLAR [8].

POLAR has the localization capability using the signal distribution (counts) among 1600 bars [9]. Since the ground calibration is just finished [10] and the in-flight calibration for 1600 bars is still on-going [11, 12], localization, spectral analysis and polarization analysis for GRBs are not finalized. We will publish these results in the forthcoming papers.

\section{POLAR in the Multi-wavelength and Multi-messenger Era}

Searching for high energy Electromagnetic (EM) counterpart is critically important for Gravitational Wave Bursts (GWBs) [13], High Energy Neutrinos (HENs) and Fast Radio Bursts (FRBs). Thanks to the unique capability of unprecedented accurate polarization measurement, a very wide FoV and high sensitivity, POLAR can play a very important role in the multi-wavelength and multimessenger era by monitoring the hard X-ray and soft gamma-ray emission from GWBs, HENs and FRBs, and providing insight to the radiation mechanism and progenitors of these celestial sources. 


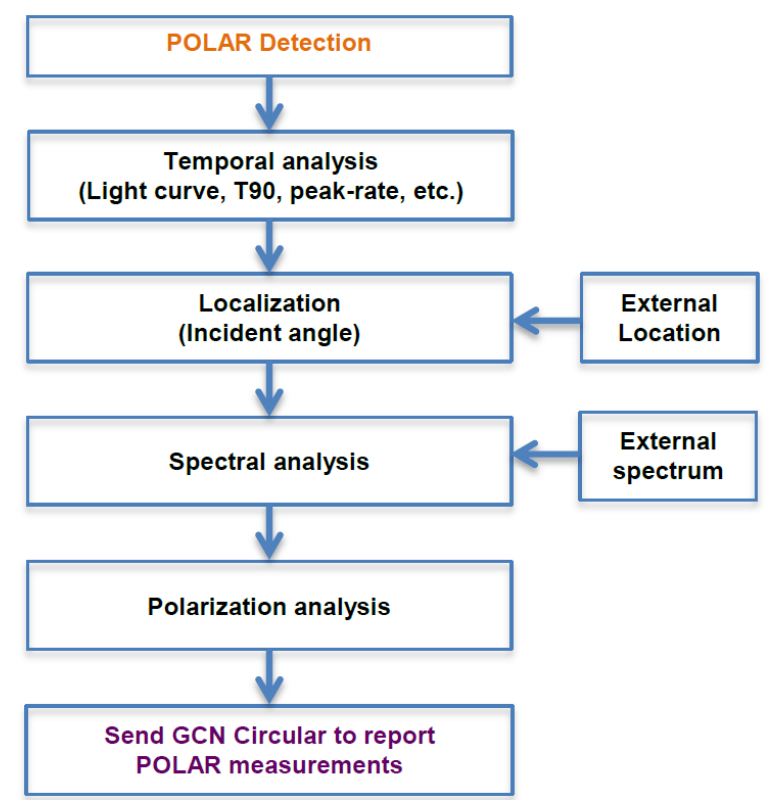

Figure 3: flow chart for GRB analysis

POLAR is originally designed to measure polarization using the Compton Scattering effect which will produce at least two hits in the detector array of 1600 bars, therefore, events with two or more hits are fully record and almost all events with only one hit are discarded. This data acquisition is called regular mode. However, recording those events just hit one bar, called singlebar mode, could significantly improve the detection sensitivity for EM counterpart, as shown in Fig. 4. Therefore, we enabled the single-bar mode once the TG2 onboard storage allows. As of Apr 1, 2017, the single-bar mode has been implemented for a total observation time of 1644 hours, as shown in Table 1.

Table 1: Summary of POLAR observation time as of Apr 1, 2017

\begin{tabular}{lcc}
\hline Items & Time (hr) & Notes \\
\hline Total time & 4567 & from Sep 22, 2016 to Apr 1,2017 \\
Time without data & 993 & instrument turned off or data lost \\
Time with data & 3574 & observation time, SAA passages included \\
Bank50 regular & 1536 & bank50 setting, regular data acquisition \\
Bank50 single-bar & 1644 & bank50 setting, single-bar data acquisition \\
Bank20 & 394 & bank20 setting, mainly for calibration \\
\hline
\end{tabular}

\section{GRB catalogue}

As of Apr 1, 2017, the time allocation of POLAR is shown in Table 1. As one can see, POLAR has taken data for a total time of 3574 hours (the normal SAA passage included). Mainly two operation settings, i.e. Bank50 and Bank20, were used, where Bank50 is for science observation and Bank20 for calibration. The total science observation time is 3180 hours, during which 55 

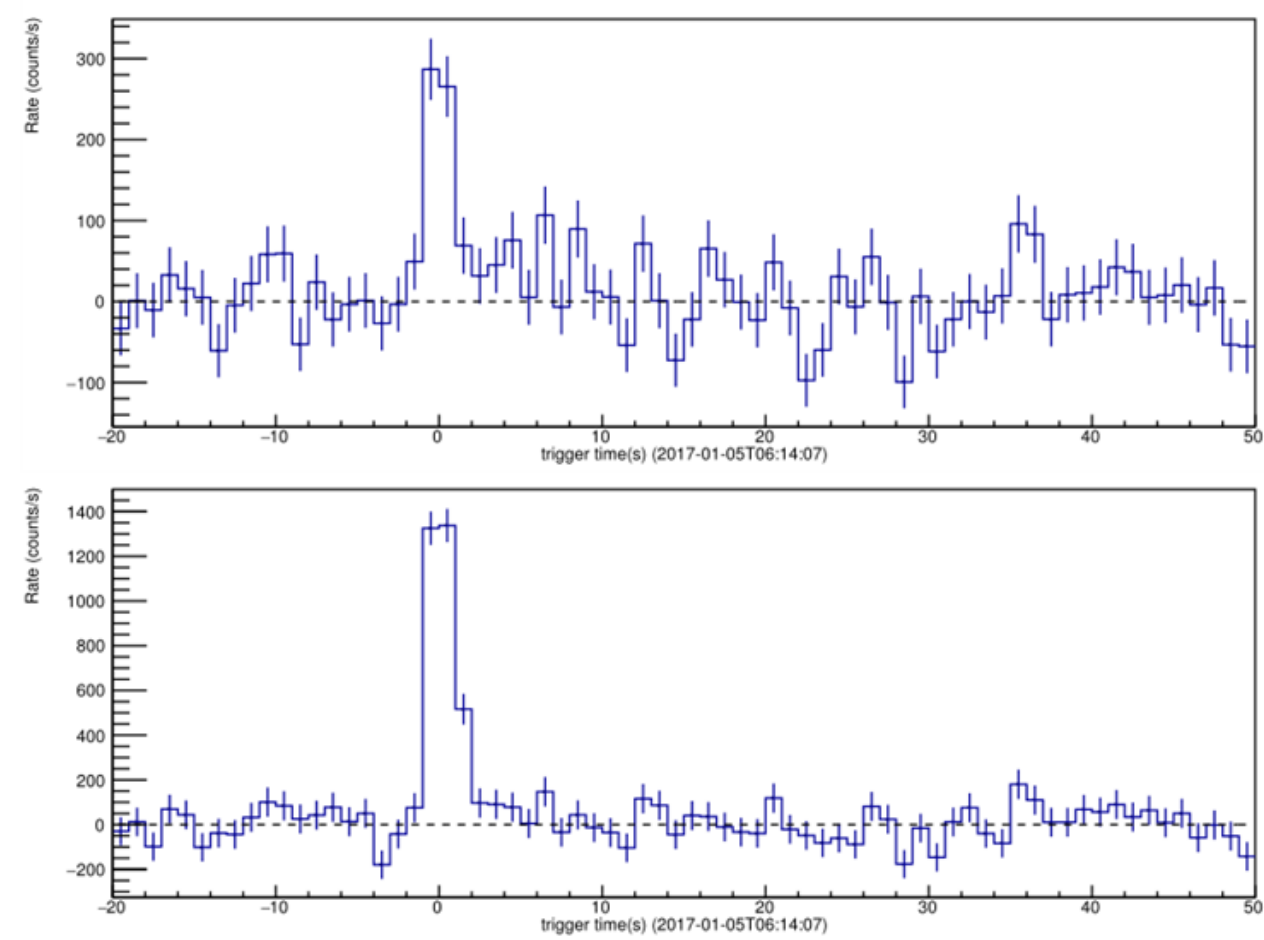

Figure 4: Light curves (backgroud-subtracted) for regular mode (top) and single-bar mode (bottom) for GRB 170105A.

GRBs were detected by POLAR together with other telescopes, for example Fermi/GBM, Swift, SPI-ACS, Konus-Wind (denoted as KW), as shown in Table 3. This corresponds to a discovery rate of about $150 \mathrm{GRBs} / \mathrm{year}$, showing that POLAR is one of the most sensitive GRB detectors in operation.

Out of these 55 GRBs, there are about 10 bright GRBs that are very suitable for polarization measurement, with the typical MDP of about $10 \%$, as shown in Table 2. We note that this is the largest sample of GRBs with high accurate polarization measurements to date, which is foreseen to shed new light on the nature of the GRB phenomenon.

Table 2: Bright GRBs detected by POLAR as of Apr 1, 2017

\begin{tabular}{lccccc}
\hline GRB Name & T90 $(\mathrm{s})$ & Total Counts & Theta $(\mathrm{deg})$ & Phi $(\mathrm{deg})$ & MDP $(1 \sigma)$ \\
\hline GRB 161218A & 6.8 & 6644 & 24.3 & 356.6 & $11 \%$ \\
GRB 161218B & 26.3 & 29340 & 77.76 & 252.2 & $12 \%$ \\
GRB 161229A & 35.77 & 35134 & 87.6 & -103.7 & $15 \%$ \\
GRB 170101A & 2.82 & 5379 & 6.04 & 72.86 & $6 \%$ \\
GRB 170114A & 8.0 & 26800 & 26.4 & 4.9 & $15 \%$ \\
GRB 170127C & 22.0 & 3600 & 41.8 & 157.6 & $13 \%$ \\
GRB 170206A & 1.2 & 12918 & 19.5 & 148.7 & $6 \%$ \\
GRB 170207A & 39.47 & 63182 & 70.6 & -2.2 & $6 \%$ \\
GRB 170210A & 67.27 & 106099 & 80.6 & 130.9 & $7 \%$ \\
GRB 170305A & 0.3 & 2400 & 31.4 & 239.1 & $10 \%$ \\
\hline
\end{tabular}




\section{Conclusion}

Since the launch on Sep 15, 2016, POLAR has detected 55 GRBs jointly with other telescopes during 3180 hours of data taken, which is equivalent to about $150 \mathrm{GRBs} / y e a r$. About 10 bright GRBs are expected to have good polarization measurements with MDP of about 10\%. After the in-flight calibration is finished, polarization analysis will be finalized. A catalogue of all POLAR GRBs will also be released at the end of the mission. In addition to the regular data acquisition mode (record events with two or more hits in 1600 bars) designed to measure the polarization, the single-bar mode (record events with one or more hits in 1600 bars) has been enabled to improve the detection sensitivity to the Electromagnetic Counterparts for Gravitational Wave Burst (GWB), Fast Radio Burst (FRB) and High Energy Neutrino (HEN) events.

\section{Acknowledgments}

This work is supported by the Strategic Priority Research Program of the Chinese Academy of Sciences (Grant No. XDB23040400). S. L. Xiong was also supported by the Hundred Talents Program of the Chinese Academy of Sciences (Grant No. 292016BRJH03).

\section{References}

[1] C. Kouveliotou, et al., ApJ 413 (1993) L101

[2] B. Zhang, Int. J. Mod. Phys. D 23 (2014) 02.

[3] M. McConnell, New Astro. Rev. 76 (2017) 1

[4] N. Produit et al., Nucl. Instr. and Meth. A 550 (2005) 616.

[5] S. Xiong et al., Nucl. Instr. and Meth. A 606 (2009) 552.

[6] M. Kole et al., ICRC Conf. Proc. 2017

[7] H. Li et al., ICRC Conf. Proc. 2017

[8] https://gcn.gsfc.nasa.gov/gcn3_archive.html

[9] E. Suarez-Garcia et al., Nucl. Instr. and Meth. A 624 (2010) 624.

[10] M. Kole, et al., submitted to Nucl. Instr. and Meth. A

[11] Y. Wang et al., ICRC Conf. Proc. 2017

[12] H. Xiao et al., ICRC Conf. Proc. 2017

[13] A. Abbott, et al., ApJL 826 (2016) 1 
Table 3: GRBs detected by POLAR as of Apr 1, 2017

\begin{tabular}{|c|c|c|c|}
\hline Number & GRB Name & Trigger time (UTC) & Joint observation \\
\hline 1 & GRB 160924A & 2016-09-24T06:04:09.040 & Fermi/GBM, SPI-ACS \\
\hline 2 & GRB 160928A & 2016-09-28T19:48:05.000 & Fermi/GBM, SPI-ACS, KW \\
\hline 3 & GRB 161009651 & 2016-10-09T15:38:07.190 & Fermi/GBM \\
\hline 4 & GRB 161011217 & 2016-10-11T05:13:44.420 & KW \\
\hline 5 & GRB 161012989 & 2016-10-12Т23:45:11.380 & KW \\
\hline 6 & GRB 161013948 & 2016-10-13T22:44:40.100 & Fermi/GBM \\
\hline 7 & GRB 161120401 & 2016-11-20Т09:38:33.520 & SPI-ACS \\
\hline 8 & GRB 161129A & 2016-11-29T07:11:40.000 & Swift/BAT, Fermi/GBM, AstroSAT \\
\hline 9 & GRB 161203A & 2016-12-03T18:41:07.750 & KW,SPI-ACS, CALET/CGBM, AstroSAT \\
\hline 10 & GRB 161205A & 2016-12-05T13:27:18.000 & Fermi/GBM \\
\hline 11 & GRB 161207A & 2016-12-07T20:42:55.000 & Fermi/GBM, CALET/CGBM \\
\hline 12 & GRB 161207B & 2016-12-07T05:22:44.000 & Fermi/GBM \\
\hline 13 & GRB 161210A & 2016-12-10T12:33:54.000 & Fermi/GBM \\
\hline 14 & GRB 161212A & 2016-12-12Т15:38:59.000 & Fermi/GBM \\
\hline 15 & GRB 161213A & 2016-12-13Т07:05:02.000 & Fermi/GBM, SPI-ACS \\
\hline 16 & GRB 161217B & 2016-12-17T03:03:44.000 & Fermi/GBM \\
\hline 17 & GRB 161217C & 2016-12-17Т03:53:15.000 & KW \\
\hline 18 & GRB 161218A & 2016-12-18T03:47:34.634 & Swift/BAT \\
\hline 19 & GRB 161218B & 2016-12-18Т08:32:41.341 & Fermi/GBM \\
\hline 20 & GRB 161219B & 2016-12-19T18:48:39.000 & Swift/BAT \\
\hline 21 & GRB 161228A & 2016-12-28T09:43:24.000 & Fermi/GBM \\
\hline 22 & GRB 161228B & 2016-12-28T13:15:40.000 & Fermi/GBM, SPI-ACS \\
\hline 23 & GRB 161228C & 2016-12-28Т00:46:20.000 & Fermi/GBM \\
\hline 24 & GRB 161229A & 2016-12-29T21:03:49.000 & Fermi/GBM \\
\hline 25 & GRB 161230A & 2016-12-30T12:16:07.000 & Fermi/GBM \\
\hline 26 & GRB 170101A & 2017-01-01T02:26:00.660 & Swift/BAT \\
\hline 27 & GRB 170101B & 2017-01-01T02:47:18.270 & Fermi/GBM \\
\hline 28 & GRB 170102A & 2017-01-02Т02:51:18.000 & KW \\
\hline 29 & GRB 170105A & 2017-01-05Т06:14:07.000 & SPI-ACS, KW \\
\hline 30 & GRB 170109A & 2017-01-09Т03:17:35.000 & Fermi/GBM \\
\hline 31 & GRB 170112B & 2017-01-12Т23:16:09.000 & Fermi/GBM \\
\hline 32 & GRB 170114A & 2017-01-14T22:01:10.000 & Fermi/GBM \\
\hline 33 & GRB 170114B & 2017-01-14T19:59:12.000 & Fermi/GBM, KW \\
\hline 34 & GRB 170120A & 2017-01-20T11:18:30.000 & Fermi/GBM \\
\hline 35 & GRB 170121A & 2017-01-21T01:36:55.200 & Fermi/GBM \\
\hline 36 & GRB 170124A & 2017-01-24T20:58:06.000 & Fermi/GBM, KW, CALET/CGBM \\
\hline 37 & GRB 170127C & 2017-01-27Т01:35:49.000 & Fermi/GBM, Fermi/LAT, AGILE, AstroSAT \\
\hline 38 & GRB 170130A & 2017-01-30Т07:14:45.000 & Fermi/GBM \\
\hline 39 & GRB 170131A & 2017-01-31T23:14:59.000 & Fermi/GBM, Swift, KW \\
\hline 40 & GRB 170202B & 2017-02-02Т07:19:54.000 & KW \\
\hline 41 & GRB 170206A & 2017-02-06T10:51:57.700 & Fermi/GBM, Fermi/LAT, SPI-ACS \\
\hline 42 & GRB 170206C & 2017-02-06T11:40:10.000 & SPI-ACS \\
\hline 43 & GRB 170207A & 2017-02-07T21:45:04.000 & Fermi/GBM, IPN, KW \\
\hline 44 & GRB 170208C & 2017-02-08T13:16:33.000 & Fermi/GBM, SPI-ACS \\
\hline 45 & GRB 170210A & 2017-02-10Т02:47:37.000 & Fermi/GBM, IPN, KW \\
\hline 46 & GRB 170219A & 2017-02-19T00:03:07.000 & Fermi/GBM, CALET/CGBM, SPI-ACS, KW, IPN \\
\hline 47 & GRB 170220A & 2017-02-20T18:48:01.000 & KW \\
\hline 48 & GRB 170228B & 2017-02-28T18:32:56.000 & Fermi/GBM \\
\hline 49 & GRB 170305A & 2017-03-05Т06:09:06.800 & Fermi/GBM, KW, SPI-ACS, Swift/BAT \\
\hline 50 & GRB 170306B & 2017-03-06T14:07:20.000 & Fermi/GBM, Fermi/LAT, SPI-ACS \\
\hline 51 & GRB 170309A & 2017-03-09T12:26:42.000 & CALET/CGBM \\
\hline 52 & GRB 170315A & 2017-03-15T13:57:53.000 & Fermi/GBM \\
\hline 53 & GRB 170317A & 2017-03-17T09:45:56.060 & Swift/BAT \\
\hline 54 & GRB 170320A & 2017-03-20Т03:42:39.000 & SPI-ACS, KW \\
\hline 55 & GRB 170325B & 2017-03-25T21:50:01.000 & KW \\
\hline
\end{tabular}

